\title{
Population Pharmacokinetics of Fludarabine in Children and Adults during Conditioning Prior to Allogeneic Hematopoietic Cell Transplantation
}

\author{
Jurgen B. Langenhorst ${ }^{1,2}$ - Thomas P. C. Dorlo ${ }^{3}$ Erik M. van Maarseveen ${ }^{4}$ Stefan Nierkens ${ }^{2}$. Jürgen Kuball ${ }^{2,5}$. \\ Jaap Jan Boelens ${ }^{1,6} \cdot$ Charlotte van Kesteren $^{1,2} \cdot$ Alwin D. R. Huitema $^{3,4}$
}

Published online: 17 October 2018

(c) The Author(s) 2018

\begin{abstract}
Background Fludarabine is often used as an important drug in reduced toxicity conditioning regimens prior to hematopoietic cell transplantation (HCT). As no definitive pharmacokinetic (PK) basis for HCT dosing for the wide age and weight range in HCT is available, linear body surface area (BSA)-based dosing is still used.

Objective We sought to describe the population PK of fludarabine in HCT recipients of all ages.

Methods From 258 HCT recipients aged 0.3-74 years, 2605 samples were acquired on days 1 (42\%), 2 (17\%), 3 (4\%) and $4(37 \%)$ of conditioning. Herein, the circulating metabolite of fludarabine was quantified, and derived concentration-time data were used to build a population PK model using non-linear mixed-effects modelling.

Results Variability was extensive where area under the curve ranged from 10 to $66 \mathrm{mg} \mathrm{h} / \mathrm{L}$. A three-compartment model with first-order kinetics best described the data. Actual body weight (BW) with standard allometric scaling was found to be the best body-size descriptor for all PK parameters. Estimated glomerular filtration rate (eGFR) was included as a descriptor of renal function. Thus, clearance was differentiated into a non-renal $(3.24 \pm 20 \% \mathrm{~L} / \mathrm{h} / 70 \mathrm{~kg})$ and renal $(\mathrm{eGFR} \times 0.782 \pm 11 \%$ $\mathrm{L} / \mathrm{h} / 70 \mathrm{~kg}$ ) component. The typical volumes of distribution of the central (V1), peripheral (V2), and second peripheral (V3) compartments were $39 \pm 8 \%, 20 \pm 11 \%$, and $50 \pm 9 \% \mathrm{~L} / 70 \mathrm{~kg}$ respectively. Intercompartmental clearances between $\mathrm{V} 1$ and $\mathrm{V} 2$, and $\mathrm{V} 1$ and $\mathrm{V} 3$, were $8.6 \pm 8 \%$ and $3.8 \pm 13 \% \mathrm{~L} / \mathrm{h} / 70 \mathrm{~kg}$, respectively.

Conclusion BW and eGFR are important predictors of fludarabine PK. Therefore, current linear BSA-based dosing leads to highly variable exposure, which may lead to variable treatment outcome.
\end{abstract}

Alwin D. R. Huitema

a.d.r.huitema-2@umcutrecht.nl

1 Pediatric Blood and Marrow Transplant Program, University

Medical Center Utrecht, Utrecht University, Utrecht,

The Netherlands

2 Laboratory of Translational Immunology, University Medical Centre Utrecht, Utrecht University, Utrecht, The Netherlands

3 Department of Pharmacy and Pharmacology, Antoni van Leeuwenhoek Hospital/Netherlands Cancer Institute, Amsterdam, The Netherlands

4 Department of Clinical Pharmacy, University Medical Centre, Utrecht, Utrecht University, Heidelberglaan 100: D.00.X, 3584 CX Utrecht, The Netherlands

5 Department of Hematology, University Medical Centre, Utrecht, Utrecht University, Utrecht, The Netherlands

6 Stem Cell Transplant and Cellular Therapies; Pediatrics, Memorial Sloan Kettering Cancer Centre, New York, NY, USA 


\section{Key Points}

Current body surface area-based dosing leads to highly variable fludarabine exposures.

All pharmacokinetic parameters were related to body weight, adequately characterized by standard allometric scaling.

Renal clearance (expressed as estimated glomerular filtration rate) corresponded to approximately $65 \%$ of total clearance for adults.

\section{Introduction}

Allogeneic hematopoietic cell transplantation (HCT) is a potentially curative treatment for a variety of malignant and benign hematological disorders. The preparative or conditioning regimen prior to HCT consists of a combination of cytotoxic agents, chemotherapy and serotherapy (antibodies against the host immune system), administered to ablate the bone marrow and the immune system [1].

Fludarabine has been evaluated in various studies as a replacement for cyclophosphamide, in combination with busulfan, aiming to decrease non-relapse mortality while maintaining the immunosuppressive and anti-cancer efficacy of cyclophosphamide [2]. Following confirmation of this hypothesis [3], fludarabine is currently used in various conditioning regimens, ranging from non-myeloablative regimens [4], to reduced intensity and myeloablative regimens.

Fludarabine is dosed based on body surface area (BSA) and intravenously administered as a monophosphate prodrug (F-ara-AMP). It is very rapidly fully converted to the circulating metabolite F-ara-A, which is distributed intracellularly. Subsequently, intracellular phosphorylation takes place to the active metabolite fludarabine triphosphate (F-ara-ATP), which is built into the DNA and RNA, thereby inhibiting DNA/RNA synthesis. This leads to apoptosis in both chronic lymphocytic leukemia cells [5] and (with different susceptibility) in cell types targeted in the HCT setting [6, 7].

As F-ara-ATP is the active metabolite, intuitively it is the form of interest for pharmacokinetic (PK) studies. However, during conditioning, only a limited number of target cells can be acquired, especially when antithymocyte globulin (ATG) is administered prior to fludarabine in the conditioning, complicating accurate quantification of intracellular F-ara-ATP. Therefore, ex vivo quantification of F-ara-ATP accumulation in pretreatment samples has been proposed in HCT recipients [8], although a relationship between this accumulation and outcome was not found [4]. Given these complexities and an apparent correlation between F-ara-A concentration and F-ara-ATP formation in cells [9], the freely circulating F-ara-A has been used primarily for PK analyses.

During the phase I trial for fludarabine, triphasic firstorder kinetics were found for F-ara-A. The main known route of elimination is through the kidney, and indeed a correlation between clearance from the central compartment and estimated glomerular filtration rate (eGFR) has been found [10].

As this study was performed in adults with chronic lymphocytic leukemia, several HCT-specific PK studies were performed to further explore fludarabine PK in this setting [9, 11-14]. However, to date, none have led to a harmonized dosing regimen for both children and adults, which takes both renal function and body size into account. Previous study results could not be extrapolated to the general population due to the use of non-population-based methods [12, 13], limited sample size [11, 14], or containing only pediatric data [9]. This causes most centers to still use BSA-based dosing, although a PK rationale for this is lacking.

Therefore, this study aims to build a population PK model, using a very large heterogeneous dataset of both pediatric and adult HCT recipients. As such, this study can provide a rational base for optimal and harmonized dosing regimens for patients of all ages in this setting, while taking renal function into account.

\section{Methods}

\subsection{Patients}

A retrospective $\mathrm{PK}$ analysis was performed with data from patients who received myeloablative conditioning before HCT, between May 2010 and January 2017, at the University Medical Centre Utrecht (UMCU), and of whom PK samples were available. No restrictions were applied for comorbidities, age, and indication for HCT. Patients were included after written informed consent was acquired. Ethical approval by the institutional Medical Ethics Committee of the UMCU was obtained under protocol number 11/063.

\subsection{Procedures}

The conditioning regimen consisted of 4 days of chemotherapy (administered from day -5 to day -2 relative to $\mathrm{HCT}$ ) consisting of a $1 \mathrm{~h}$ infusion of fludarabine phosphate directly followed by a $3 \mathrm{~h}$ infusion of busulfan (Busilvex; Pierre Fabre, Paris, France). A 1 h infusion of clofarabine 
preceded fludarabine infusion in children with malignancies. Rabbit ATG was added in the unrelated donor HCT setting: 4-h infusions on 4 consecutive days from day $-9(10 \mathrm{mg} /$ $\mathrm{kg}<30 \mathrm{~kg}, 7.5 \mathrm{mg} / \mathrm{kg}>30 \mathrm{~kg}$ ) for children, and four 12-h infusions from day $-12(6 \mathrm{mg} / \mathrm{kg})$ for adults.

Patients received either a cumulative dose of $160 \mathrm{mg} / \mathrm{m}^{2}$ of fludarabine phosphate or $40 \mathrm{mg} / \mathrm{m}^{2}$ fludarabine phosphate combined with $120 \mathrm{mg} / \mathrm{m}^{2}$ clofarabine. Intravenous busulfan was targeted to a myeloablative cumulative 4-day exposure of $90 \mathrm{mg} \mathrm{h} / \mathrm{L}$ or $30 \mathrm{mg} \mathrm{h} / \mathrm{L}$ for Fanconi anemia patients (expressed as area under the curve for all doses $\left[\mathrm{AUC}_{\mathrm{T} 0-\infty}\right]$ ). For patients receiving ATG, clemastine, paracetamol, and $2 \mathrm{mg} / \mathrm{kg}$ prednisolone (with a maximum of $100 \mathrm{mg}$ ) were administered intravenously prior to ATG infusion.

\subsection{Pharmacokinetic (PK) Samples and Analyses}

Concentrations of the circulating metabolite of fludarabine (F-ara-A, hereafter referred to as fludarabine) were analyzed in PK samples taken for routine busulfan therapeutic drug monitoring (TDM) according to local protocol. Quantification of fludarabine concentrations was performed using a liquid chromatography mass spectrometry method validated according to US FDA and European Medicines Agency (EMA) guidelines as described previously, with a lower limit of quantification of $0.001 \mathrm{mg} / \mathrm{L}$ [15]. In the TDM protocol, plasma samples were drawn on the first or second day of conditioning. If considered necessary for TDM purposes, samples were also drawn on the following days. Additional samples were taken on the final day of conditioning. In general, plasma samples were taken at 4, 5, 6, and $7 \mathrm{~h}$, after the end of fludarabine infusion. For a subset of patients, additional samples were collected from 7 to $24 \mathrm{~h}$ post-infusion. From January 2016 onwards, additional samples were collected between the end of fludarabine infusion and the start of busulfan infusion, 15-45 min after the end of fludarabine infusion.

A population approach based on non-linear mixed-effects modeling was applied [16], using the software package NONMEM version 7.3.0 (Icon, Hanover, MD, USA). Pirana version 2.9.5 and $\mathrm{R}$ version 3.3.3 were used for workflow management, and data handling and visualization, respectively $[17,18]$. The first-order conditional estimation option with interaction between random and residual error components (FOCE-I), as implemented in NONMEM, was used as the estimation method.

\subsection{Pharmacokinetic (PK) Model-Building Procedure}

For the structural model, one-, two- and three-compartment models with first-order kinetics were tested.
Interindividual variability (IIV) was assumed to follow a log-normal distribution and was therefore implemented into the model as follows:

$P_{i}=P_{\mathrm{pop}} \times e^{\left(\eta_{i}\right)}$

where $\mathrm{P}_{\mathrm{i}}$ depicts the individual or post hoc value of the parameter for the $i$ th individual, $P_{\text {pop }}$ depicts the population mean for the parameter, and $\eta_{i}$ predicts the empirical Bayes estimate of IIV for the $i$ th individual, sampled from a normal distribution with a mean of zero and a variance of $\omega^{2}$.

Interoccasion variability (IOV) was implemented similarly, with each dose and subsequent sampling defined as a separate occasion. This variability was evaluated for all parameters to diagnose potential time-dependent trends and to allow for random unaccounted variability between dosing moments.

Residual error was evaluated as a proportional or additive error, or as a combination of both (Eq. 2).

$P_{\mathrm{obs}}=P_{i} \times\left(1+\varepsilon_{\text {proportional }}\right)+\varepsilon_{\text {additive }}$

where $P_{\text {obs }}$ is the observed value, $\varepsilon_{\text {proportional }}$ is the proportional error component, and $\varepsilon_{\text {additive }}$ is the additive error component. Residual error components are sampled from a normal distribution with a mean of zero and variance of $\sigma$.

\subsection{Covariate Model}

Following development of the structural and stochastic PK model, potential predictors (covariates) for variability in PK parameters were evaluated. Assessed covariates included patient-related (body size, i.e. actual body weight [BW], fat-free mass [FFM], BSA, and other, i.e. age, renal function) and treatment-related (serotherapy, additional coconditioning agents) factors. FFM was calculated using the equation developed by McCune et al. (Eq. 3) [19], and BSA was calculated according to the method developed by Du Bois et al. [20].

$P_{i}=9270 \times\left(\left(\frac{\mathrm{BW}}{\frac{\mathrm{BW}}{\mathrm{HT}^{2}} \times S_{\mathrm{sex}}+C_{\mathrm{sex}}}\right)\right)$

where HT corresponds to height in meters, BW corresponds to actual BW in $\mathrm{kg}$, and $S_{\text {sex }}\left(\mathrm{m}^{2} / \mathrm{kg}\right)$ and $C_{\text {sex }}$ (dimensionless) are constants that change upon sex. $S_{\text {sex }}$ takes values of 216 and 244, and $C_{\text {sex }}$ takes values of 6680 and 8780, for males and females, respectively.

Continuous covariates were evaluated using both a linear function and a power function (Eqs. 4 and 5):

$P_{i}=P_{\text {pop }} \times\left(\left(\frac{\operatorname{Cov}_{i}}{\operatorname{Cov}_{\text {typical }}}\right) \times 1\right)$ 
$P_{i}=P_{\mathrm{pop}} \times\left(\frac{\operatorname{Cov}_{i}}{\operatorname{Cov}_{\text {typical }}}\right)^{p}$

where $\operatorname{Cov}_{\mathrm{i}}$ is the covariate value for the $i$ th individual, and $\operatorname{Cov}_{\text {typical }}$ is the typical or median value for the covariate in the population. The estimated parameters are $l$ and $p$ for the linear and power function, respectively.

Binary categorical covariates were tested by using Eq. 6 :

$P_{i}=P_{\text {pop }} \times\left(1+P_{\text {cov }}\right)$

where $P_{\text {cov }}$ is the estimated proportional factor with which the parameter changes for a specific covariate value.

To implement body size descriptors on PK parameters, standard allometric scaling was initially applied using Eq. 4, with $p$ fixed at 0.75 (BW/FFM) or 1 (BSA) for clearances, and 1 for distribution volumes (BW/FFM/BSA). Alternative body size measures (FFM, BSA) were tested as a replacement of BW. Empirical estimation of the exponents was tested for the optimal body size descriptor and was only preferred, if this resulted in a relevant improvement of the model fit and when the estimated parameters were markedly different from the theoretical values.

Renal function was evaluated as a covariate, since fludarabine is predominantly eliminated renally $[10,21]$. As creatinine levels were not measured daily, the mean value of available individual creatinine values between day -7 and day 0 prior to infusion were used. Subsequently, renal function (as eGFR) was calculated using the Cockroft-Gault equation, which takes age into account [22]. eGFR for patients below the age of 17 years for women and 14 years for men was calculated using the Schwartz equation [23]. To prevent physiologically implausible high eGFR values, these were capped to a maximum. Maximum eGFR was set at $140 \mathrm{~mL} / \mathrm{min} / 1.73 \mathrm{~m}^{2}$, but was assumed to increase to this value from birth until 1.5 years of age, starting at $35 \mathrm{~mL} /$ $\mathrm{min} / 1.73 \mathrm{~m}^{2}$ (25\% of maximum value), and to decline by $8 \mathrm{~mL} / \mathrm{min} / 1.73 \mathrm{~m}^{2}$ per decade after the age of 30 years, as suggested earlier [24].

\subsection{PK Model Evaluation}

The structural and covariate model with corresponding estimates had to be scientifically and biologically plausible. To investigate parameter-covariate relationships, covariates were plotted versus empirical Bayes estimates of IIV. Trends in these plots indicated potential relationships.

The addition of a parameter had to result in a significant improvement in model fit. This was evaluated using the objective function value (OFV), equal to minus twice the log-likelihood, which is assumed to follow a Chisquare distribution. In hierarchical models, an OFV change $(\Delta \mathrm{OFV})$ of -3.84 corresponds to a $p$ value of 0.05 for the addition of one parameter (i.e. 1 degree of freedom). Covariates were evaluated for significance using forward inclusion and backward elimination [12]. A significance level of $p<0.005(-7.9 \Delta \mathrm{OFV})$ was used for the forward inclusion, and $p<0.001(-10.8 \Delta \mathrm{OFV})$ for backward elimination. In addition, inclusion of a covariate had to result in a substantial decline in unexplained IIV [25].

A visual inspection of model performance was performed through standard goodness-of-fit plots. Examples of these plots are observed concentrations plotted versus individual and population- predicted concentrations, and conditional weighted residuals (CWRES) versus time and observed concentrations [26]. Particular emphasis was given to goodnessof-fit plots stratified for age, to assess potential age-related misspecification.

Furthermore, relative standard errors (RSEs, as estimated from the \$COVARIANCE step) of all parameters, and shrinkage of random and residual error components, were assessed [12]. Values below 30\% for shrinkage (IIV and residual error) and RSE (all parameters) were deemed acceptable. Finally, the condition number was calculated after each addition of a parameter, to check for over-parameterization, where a value below 1000 was accepted [27].

Several evaluation techniques were performed, all in accordance with EMA and FDA guidelines for population PK analyses [28, 29]. A non-parametric bootstrap evaluation (1000 samples) was performed to assess parameter precision. In addition, the normalized prediction distribution error (NPDE) was evaluated. Discrepancies between the final model and 1000 simulations of the model were evaluated, taking into account the correlation between observations in the same individual and the predictive distribution [30].

To assess the simulation properties, prediction-corrected visual predictive checks (VPCs) were created to assess the predictive performance of the final model compared with the observed concentrations. The prediction-corrected VPC allows for variability in dosing [31]. In this analysis, the observed concentration data, and its median and 95\% confidence intervals (CIs), were compared with the $95 \%$ CIs of the predicted mean, 2.5th and 95th percentile, derived from 1000 model simulations.

\section{Results}

\subsection{Patients and Samples}

A total of 258 patients with a median age of 18 years were included in this study. Of these patients, 197 received a cumulative fludarabine dose of $160 \mathrm{mg} / \mathrm{m}^{2}$, and 61 received a cumulative dose of $40 \mathrm{mg} / \mathrm{m}^{2}$ in combination with 
Table 1 Patient characteristics

Children and adolescents $\quad$ Adults $(>20$ years $) \quad$ Total

$(\leq 20$ years $)$

\begin{tabular}{|c|c|c|c|}
\hline \multicolumn{4}{|l|}{ Available data } \\
\hline No. of patients $[n(\%)]^{\mathrm{a}}$ & $134(52)$ & $124(48)$ & $258(100)$ \\
\hline Total no. of pharmacokinetic samples $[n(\%)]^{\mathrm{a}}$ & $1384(53)$ & $1221(47)$ & $2605(100)$ \\
\hline No. of samples per patient ${ }^{b}$ & $10(3-19)$ & $10(3-16)$ & $10(3-19)$ \\
\hline \multicolumn{4}{|l|}{ Patient characteristics } \\
\hline Female sex $[n(\%)]^{\mathrm{c}}$ & $52(39)$ & $46(37)$ & $98(38)$ \\
\hline Age at transplantation, years ${ }^{\mathrm{d}}$ & $9.6(0.2-20,2.7-14)$ & $54(22-74,40-62)$ & $18(0.2-74,40-62)$ \\
\hline Actual bodyweight, $\mathrm{kg}^{\mathrm{d}}$ & $29(4.3-96,14-52)$ & $78(47-130,65-89)$ & $60(4.3-130,28-78)$ \\
\hline Renal function ${ }^{\mathrm{d}}$ [eGFR: $\left.\mathrm{mL} / \mathrm{min} / 1.73 \mathrm{~m}^{2}\right]^{\mathrm{d}}$ & $140(40-140,110-140)$ & $110(25-140,93-130)$ & $120(25-140,100-140)$ \\
\hline \multicolumn{4}{|l|}{ Indication for transplantation $[n(\%)]^{\mathrm{c}}$} \\
\hline Benign disorder & $65(49)$ & $4(3.2)$ & $69(27)$ \\
\hline Leukemia & $69(51)$ & $48(39)$ & $117(45)$ \\
\hline Lymphoma & $0(0)$ & $17(14)$ & $17(6.6)$ \\
\hline Myelodysplastic syndrome & $0(0)$ & $32(26)$ & $32(12)$ \\
\hline Plasma cell disorder & $0(0)$ & $23(19)$ & $23(8.9)$ \\
\hline \multicolumn{4}{|l|}{ Transplant cell source $[\text { no. }(\%)]^{\mathrm{c}}$} \\
\hline Peripheral blood & $2(2)$ & $109(88)$ & $111(43)$ \\
\hline Cord blood & $94(70)$ & $12(10)$ & $106(41)$ \\
\hline Bone marrow & $38(28)$ & $3(2)$ & $41(16)$ \\
\hline
\end{tabular}

$e G F R$ estimated glomerular filtration rate

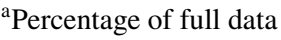

${ }^{\mathrm{b}}$ Data are expressed as median (range)

${ }^{c}$ Percentage of subgroup data within selected population (adults/children/total)

${ }^{\mathrm{d}}$ Data are expressed as median (range, interquartile range)

${ }^{\mathrm{e}}$ Calculated as described in the Methods, Sect. 2.5

clofarabine. Of the obtained 2605 samples, none were below the lower limit of quantification. The concentration-time data were divided over 596 administered doses, of which $116(19 \%)$ contained peak samples $(<3 \mathrm{~h})$ and $117(20 \%)$ contained trough samples $(>8 \mathrm{~h})$. Detailed patient characteristics are shown in Table 1.

\subsection{Structural and Stochastic Model}

BW was a priori included as a covariate using a power function (Eq. 4) on all (intercompartmental) clearances and volumes of distribution during model development. The exponents for BW on volume of distribution and clearance were fixed to 1.0 and 0.75 , respectively, prior to covariate analyses.

A three-compartment model best described the data. In addition, both the VPCs and goodness-of-fit plots showed substantial misspecification for the two-compartment model, which was absent in the three-compartment model. The three-compartment model was parameterized in terms of volume of distribution of the central (V1), peripheral (V2), and second peripheral (V3) compartment, and clearance from the central compartment as well as intercompartmental clearance between V1 and V2 (Q2) and V1 and V3 (Q3).

IIV was added on $\mathrm{V} 1$ as well as clearance from the central compartment, and inclusion of IOV was also significant for both these parameters. Inclusion of IOV and IIV on Q2, Q3, V2, and V3, led to improved model fit, however this model was highly over-parameterized (condition number $>1000$ ) and unstable (sensitive to initial estimates). Upon visual inspection of the random effects and estimation of the covariance matrices, it was shown that the random effects on volume (V1, V2, V3) and clearances $(\mathrm{Cl}, \mathrm{Q} 2, \mathrm{Q} 3)$ were highly correlated. Therefore, single random effects (both IIV and IOV) were estimated for V1, V2, and V3, and for CL, $\mathrm{Q} 2, \mathrm{Q} 3$, respectively. This approximation was adequate to describe the observed variability and provided stable and reproducible parameter estimates (Table 2).

\subsection{Covariate Model}

Figure $1 \mathrm{a}$ and $\mathrm{b}$ depict the variability in observed fludarabine concentrations over time (Fig. 1a), and total exposure 
Table 2 Final population pharmacokinetic parameter estimates
Estimate $\left(95 \% \mathrm{CI}^{\mathrm{a}}\right) \quad$ Shrinkage

\begin{tabular}{|c|c|c|c|c|}
\hline \multicolumn{5}{|l|}{ Structural model } \\
\hline \multicolumn{5}{|c|}{$\mathrm{Cl}=\left(\mathrm{Cl}_{70 \mathrm{~kg}-\text { non-renal }}+\mathrm{eGFR} \times\right.$ Slope $\left._{\text {pop }}\right) \times\left(\frac{\mathrm{BW}}{70 \mathrm{~kg}}\right)^{0.75}$} \\
\hline $\mathrm{Cl}_{70 \text { kg-non-renal }}(\mathrm{L} / \mathrm{h})$ & \multicolumn{2}{|l|}{$3.2(1.6-4.9)$} & 3.2 & $2.0-4.3$ \\
\hline Slope $_{\text {pop }}$ & \multicolumn{2}{|l|}{$0.78(0.57-1.0)$} & 0.79 & $0.65-0.95$ \\
\hline \multicolumn{5}{|l|}{$V_{1}=V_{1,70 \mathrm{~kg}} \times\left(\frac{\mathrm{BW}}{70 \mathrm{~kg}}\right)^{1}$} \\
\hline$V_{1,70 \mathrm{~kg}}(\mathrm{~L})$ & \multicolumn{2}{|l|}{$39(33-45)$} & 39 & $33-46$ \\
\hline \multicolumn{5}{|l|}{$V_{2, \mathrm{pop}}=V_{2,70 \mathrm{~kg}} \times\left(\frac{\mathrm{BW}_{\mathrm{i}}}{70 \mathrm{~kg}}\right)^{1}$} \\
\hline$V_{2,70 \mathrm{~kg}}(\mathrm{~L})$ & \multicolumn{2}{|l|}{$20(17-24)$} & 21 & $16-28$ \\
\hline \multicolumn{5}{|l|}{$V_{3}=V_{3,70 \mathrm{~kg}} \times\left(\frac{\mathrm{BW}}{70 \mathrm{~kg}}\right)^{1}$} \\
\hline$V_{3,70 \mathrm{~kg}}(\mathrm{~L})$ & \multicolumn{2}{|l|}{$50(41-58)$} & 51 & $43-64$ \\
\hline \multicolumn{5}{|l|}{$Q_{2}=Q_{2,70 \mathrm{~kg}} \times\left(\frac{\mathrm{BW}}{70 \mathrm{~kg}}\right)^{0.75}$} \\
\hline$Q_{2,70 \mathrm{~kg}}(\mathrm{~L} / \mathrm{h})$ & \multicolumn{2}{|l|}{$8.6(6.8-10)$} & 8.8 & $7.3-11$ \\
\hline \multicolumn{5}{|l|}{$Q_{3}=Q_{3,70 \mathrm{~kg}} \times\left(\frac{\mathrm{BW}_{\mathrm{i}}}{70 \mathrm{~kg}}\right)^{0.75}$} \\
\hline$Q_{3,70 \mathrm{~kg}}(\mathrm{~L} / \mathrm{h})$ & \multicolumn{2}{|l|}{$3.8(2.9-4.6)$} & 3.7 & $2.4-5.0$ \\
\hline \multicolumn{5}{|l|}{ Random variability } \\
\hline Interindividual variability on $\mathrm{CL}, Q 2, Q 3(\%)$ & $23(15-31)$ & $7 \%$ & 23 & $20-26$ \\
\hline Interindividual variability on $V 1, V 2, V 3(\%)$ & $48(36-60)$ & $15 \%$ & 47 & $37-57$ \\
\hline Interoccasion variability on $\mathrm{CL}, Q 2, Q 3(\%)$ & $12(9.6-14)$ & $28 \%$ & 12 & $11-14$ \\
\hline Interoccasion variability on $V 1, V 2, V 3(\%)$ & $31(18-44)$ & $37 \%$ & 32 & $25-38$ \\
\hline Proportional residual error $(\%)$ & $6.3(4.3-8.3)$ & $20 \%$ & 6.2 & $5.4-7.2$ \\
\hline
\end{tabular}

$C I$ confidence interval, pop population, $i$ individual, $B W$ actual body weight, $V_{l}$ central volume of distribution, $V 2$ peripheral volume of distribution, $V 3$ second peripheral volume of distribution, $Q 2$ intercompartmental clearance V1-V2, Q3 intercompartmental clearance V1-V3

Population estimates $V_{1,70 \mathrm{~kg}}, V_{2,70 \mathrm{~kg}}, V_{3,70 \mathrm{~kg}}, \mathrm{Cl}_{70 \mathrm{~kg}}, \mathrm{Q}_{2,70 \mathrm{~kg}}, \mathrm{Q}_{3,70 \mathrm{~kg}}$ correspond to a subject weighing $70 \mathrm{~kg}$ and are adjusted to an individual value, according to the corresponding parameter formula in the table

${ }^{a}$ Calculated from the residual standard error estimated from the covariance step

${ }^{b}$ Based on 1000 bootstrap samples, of which $95 \%$ were successful

(observed $\mathrm{AUC}_{\mathrm{T} 0-\infty}$ ) (Fig. 1b) stratified BSA-adjusted dose $\left(10\right.$ and $\left.40 \mathrm{mg} / \mathrm{m}^{2}\right)$. In both the low $\left(40 \mathrm{mg} / \mathrm{m}^{2}\right)$ and high $\left(160 \mathrm{mg} / \mathrm{m}^{2}\right)$ dose groups, concentrations over time after dose were highly variable, resulting in a large range for $\mathrm{AUC}_{\mathrm{T} 0-\infty}(2.7-12,10-66 \mathrm{mg} \mathrm{h} / \mathrm{L}$ for 10 and $40 \mathrm{mg} /$ $\mathrm{m}^{2}$, respectively). With a median $\mathrm{AUC}_{\mathrm{T} 0-\infty}$ of $21 \mathrm{mg} * \mathrm{~h} / \mathrm{L}$ (range 5.7-42) and $26 \mathrm{mg} \mathrm{h} / \mathrm{L}$ (range 13-65) at a $40 \mathrm{mg} /$ $\mathrm{m}^{2}$ dose for children and adults, respectively, these values are slightly higher than those reported by Ivaturi et al. for children (median $18 \mathrm{mg} \mathrm{h} / \mathrm{L}$ ) and by Long-Boyle et al. for adults (median $20 \mathrm{mg} \mathrm{h} / \mathrm{L}$ ) at the same cumulative dose of $160 \mathrm{mg} / \mathrm{m}^{2}$.

Figure $1 \mathrm{c}$ depicts the exposures at different weight quartiles. Low weights correlate to low exposures and high weights correlate to high exposures, indicating that BSA is not a sufficient body-size descriptor for fludarabine clearance. Figure 1d depicts exposures at subgroups stratified for eGFR values according to the classification of the National Kidney Foundation [32]: healthy renal function (eGFR $>90 \mathrm{~mL} / \mathrm{min} / 1.73 \mathrm{~m}^{2}$ ), mild (eGFR $60-90 \mathrm{~mL} /$ $\left.\min / 1.83 \mathrm{~m}^{2} ; n=37\right)$ and moderate (eGFR $30-60 \mathrm{~mL} /$ $\min / 1.83 \mathrm{~m}^{2} ; n=11$ ) renal impairment [6]. Healthy renal function was further subdivided into above $(n=129)$ and below $(n=81)$ the median (eGFR $120 \mathrm{~mL} / \mathrm{min} / 1.73 \mathrm{~m}^{2}$ ). Herein, it seems that eGFR and fludarabine clearance are correlated.

Therefore, non-renal clearance was differentiated from renal clearance by adding eGFR with an estimated slope. 

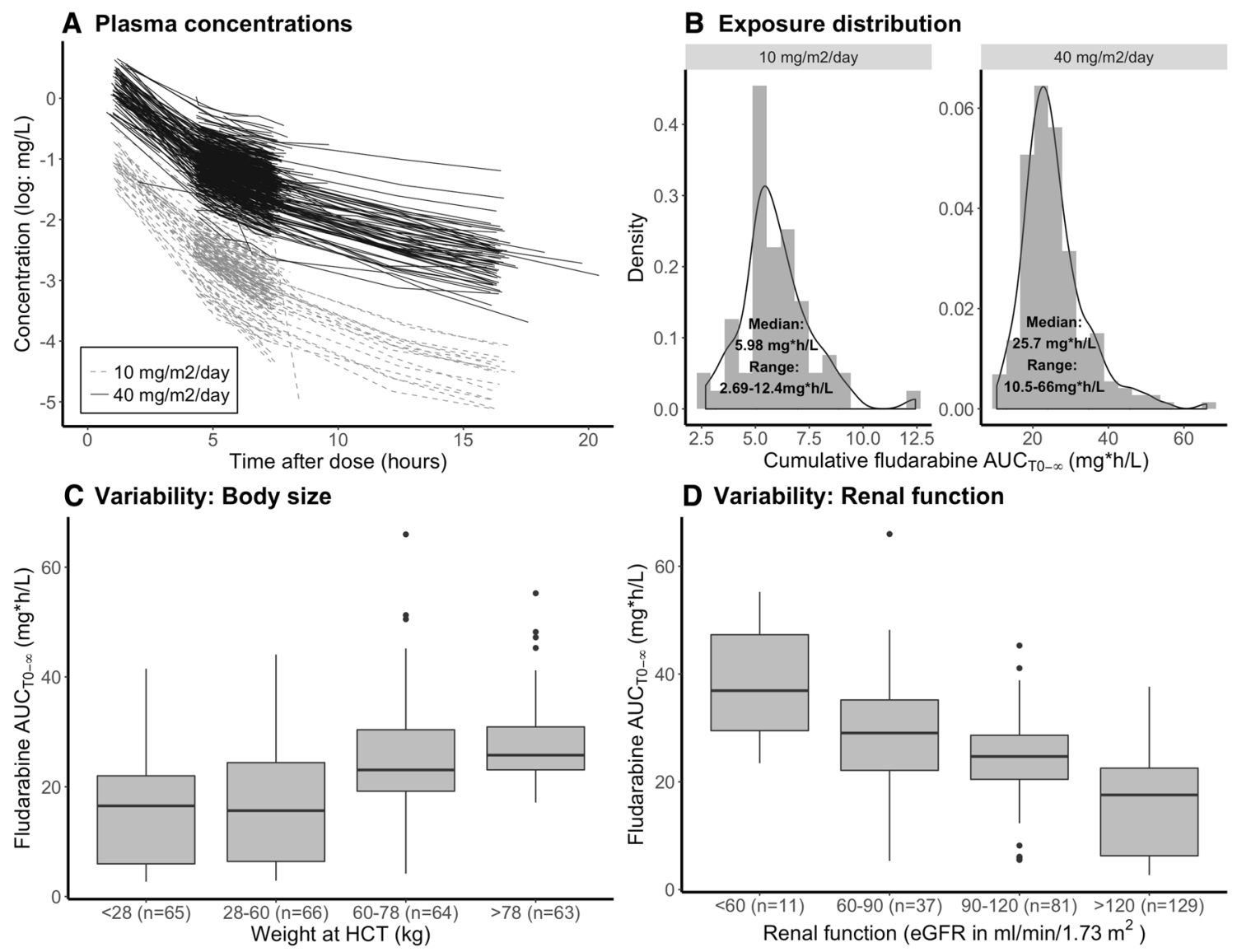

Fig. 1 Exposure variability and covariates predicting variability. a Fludarabine plasma concentrations versus time after last dose on a logarithmic scale. Each line corresponds to a single dose, stratified to dose. b Histogram (grey area) and density plot (black solid line) of the observed $\mathrm{AUC}_{\mathrm{T} 0-\infty} . \mathrm{AUC}_{\mathrm{T} 0-\infty}$ of patients receiving a low dose

BW and IIV were implemented on the total clearance [33], as illustrated in Eq. 7:

$\mathrm{Cl}_{i}=\left(\mathrm{Cl}_{70 \mathrm{~kg}-\text { non-renal }}+\mathrm{eGFR} \times\right.$ Slope $\left._{\mathrm{pop}}\right) \times\left(\frac{\mathrm{BW}}{70 \mathrm{~kg}}\right)^{0.75} \times \mathrm{e}^{\mathrm{IIV}}$

where IIV represents the IIV for clearance, Q2 and Q3; eGFR is based on individual creatinine levels and used in this equation as $1 / \mathrm{h} / \mathrm{kg}$; and Slope $\mathrm{pop}_{\text {po }}$ is a unitless estimate, representing the fraction of eGFR accounting for renal clearance of fludarabine.

The addition of eGFR resulted in a statistically significant improvement in fit $(\triangle \mathrm{OFV}-172,1$ degree of freedom, $p<0.001$ ) and IIV on clearance reduced from 34 to $23 \%$. The Slope $_{\text {pop }}$ was estimated at 0.78 (RSE 11\%), indicating that there is limited renal resorption of fludarabine.

The use of alternative body size descriptors FFM and BSA, did not improve the model fit $(\Delta \mathrm{OFV}$ of +60 and +68 , respectively). Estimation of the allometric exponents for volume or clearance resulted in values very close to 0.75 and

$\left(40 \mathrm{mg} / \mathrm{m}^{2}\right)$ were normalized to $160 \mathrm{mg} / \mathrm{m}^{2}$. c Boxplots of $\mathrm{AUC}_{\mathrm{T} 0-\infty}$ per weight quartile of observed $\mathrm{AUC}_{\mathrm{T} 0-\infty}$. d Boxplots of observed $\mathrm{AUC}_{\mathrm{T} 0-\infty}$ stratified for renal function. $H C T$ hematopoietic cell transplantation, $e G F R$ estimated glomerular filtration rate

1.0 , respectively, and did not result in a relevant improvement in model fit. Therefore, the fixed exponents were kept in the model. After inclusion of BW and eGFR, no trends were visible in the plots of empirical Bayes estimates of IIVs versus eGFR and BW.

No other covariates, such as coadminstration of clofarabine or rabbit ATG, could be identified. Importantly, no effect of age could be identified on any of the PK parameters.

\subsection{Model Evaluation}

The final estimates and the results of the bootstrap analysis are shown in Table 2. The median, 2.5th and 97.5th percentiles of bootstrap estimates are in line with those of the original data.

Age- and renal function-stratified goodness-of-fit plots (Fig. 2) generally demonstrated accurate population and individual predictions. Population predictions for children with a renal function below $60 \mathrm{~mL} / \mathrm{min} / 1.73 \mathrm{~m}^{2}$ seemed a bit off, although this group consisted of only four patients. 


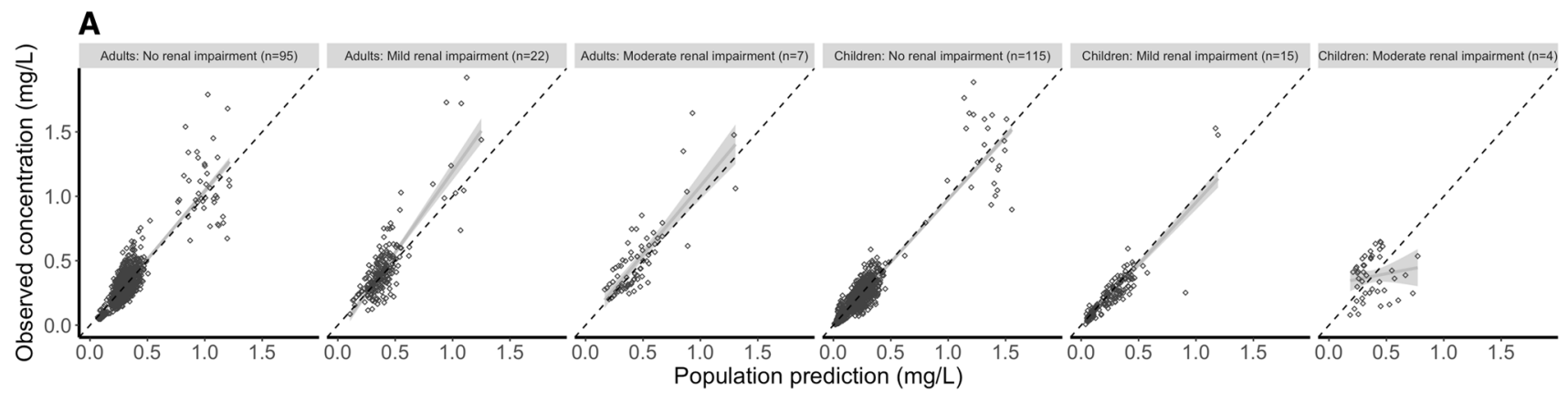

B

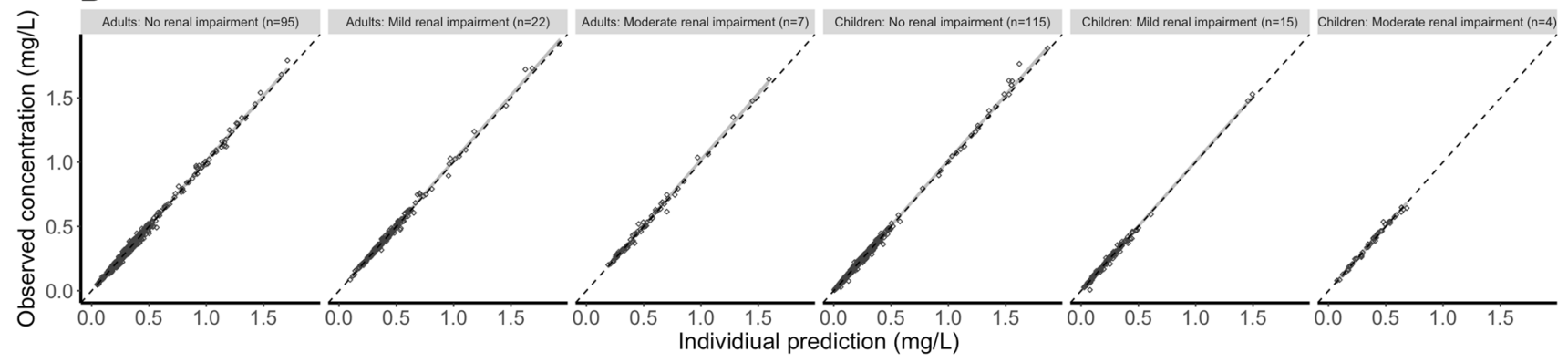

Fig. 2 Goodness-of-fit plots for the final pharmacokinetic model stratified by age. a, b Depict the population and individual predictions, respectively, versus observed concentrations, stratified for age $(</ \geq 20$ years) and renal function (eGFR $>90,90-60,<60 \mathrm{~mL} /$ $\min / 1.73 \mathrm{~m}^{2}$ ). Black open circles represent the observations and the solid grey line is a local regression fit of these values. Dashed lines depict the line of unity. $e G F R$ estimated glomerular filtration rate
No trends were observed for CWRES versus time after dose (Fig. 3a), predicted concentrations (Fig. 3b), or covariates of renal function (Fig. 3c) and weight (Fig. 3d). The NPDE analysis showed a normal distribution, and no trends were observed in the NPDEs versus time or predictions (data not shown). The VPC showed that the median and 95\% CI of the observed data were in line with those from the simulationbased predictions from the model for all strata (Fig. 4), but not the children with moderate renal impairment $(<60 \mathrm{~mL} /$ $\min / 1.73 \mathrm{~m}^{2}$ ). The median of the observations is slightly higher than predicted, indicating an over-prediction of clearance for this group. All four children in this group were at an age $<0.5$ years, indicating that maturation for very young children is possibly not well accounted for in the model.

We performed a sensitivity analysis to investigate potential bias caused by the imbalanced sampling design. This analysis showed that bias in clearance was negligible (data not shown).

\section{Discussion}

To our knowledge, this is the first PK model developed on the basis of a large and diverse dataset $(n=258)$ including both adult and pediatric patients. In addition, this study covered the vast majority of HCT indications: acute leukemia, lymphoma, plasma cell disorders, myelodysplastic syndrome, and a variety of benign disorders (autoimmune diseases, immune deficiencies, bone marrow failure, and metabolic diseases). This allowed for a unique platform to quantify fludarabine PK for all HCT populations.

Fludarabine PK was best described using a three-compartment model, which is in line with the data of the phase I study [10]. In contrast, fludarabine plasma PK in two other population PK studies was described using a two-compartment model $[9,14]$; however, these analyses had smaller sample sizes $(n=54 / n=133)$ and included only children. In addition, no formal testing of three-compartmental kinetics was mentioned.

Allometric scaling of all parameters using BW was found to best account for differences in body size, and, after inclusion of eGFR, no body size-independent effect of age could be identified. Other studies did not compare body-size descriptors but rather implemented either allometric scaling to BW [9] or BSA-adjusted PK [11, 14] a priori. Given the evidence supporting allometric theory over BSA-adjusted PK [34, 35], allometry-based adjustment is preferred. In addition, we found that fludarabine BSA-based dosing led to major under- and over-prediction of exposures at high and low BW, respectively.

Similar to Ivaturi et al., eGFR was included using a body size-adjusted method. This method has the advantage of reflecting solely renal function, while absolute eGFR 

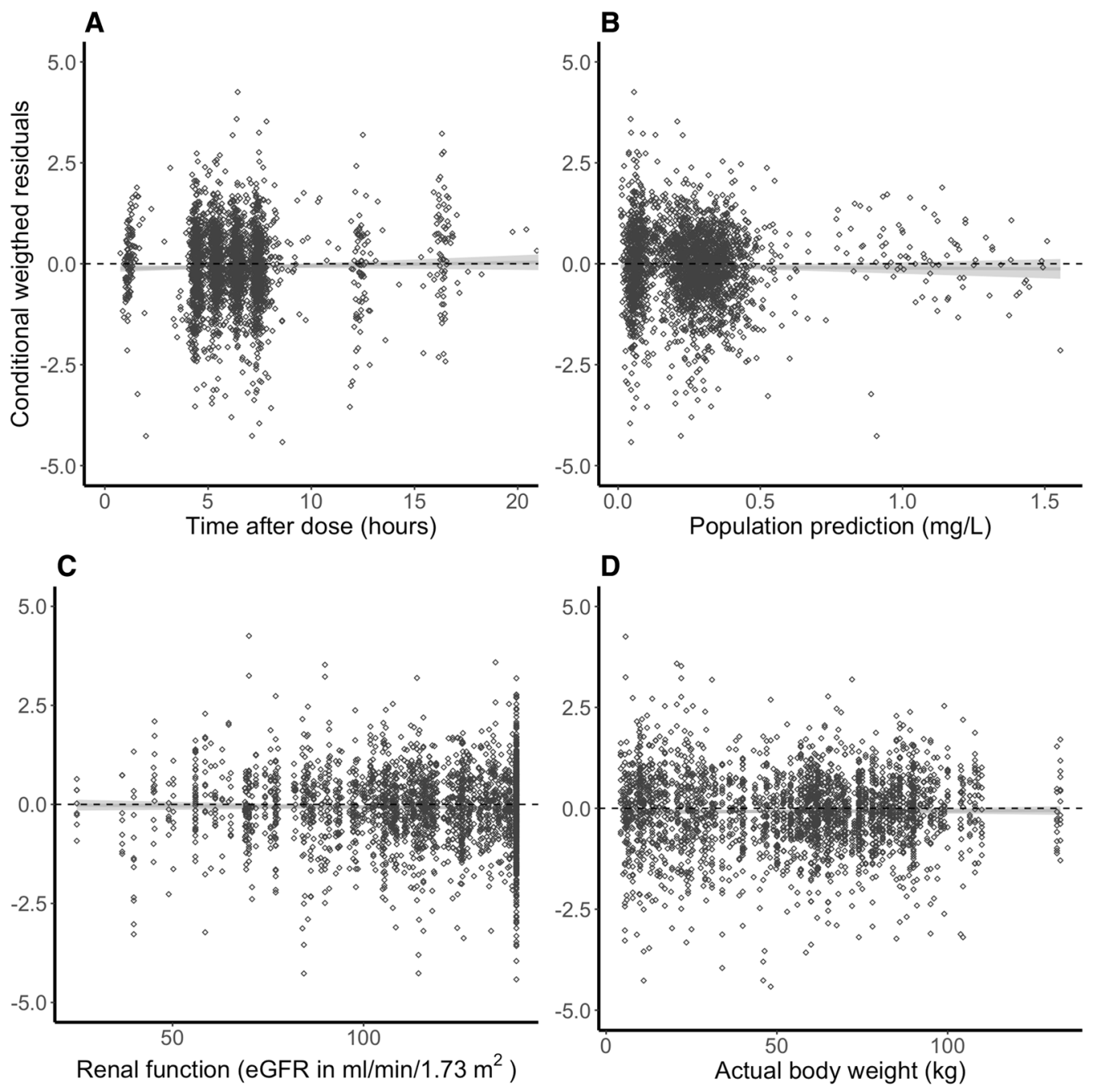

Fig. 3 Conditional weighted residuals versus time after dose, population predictions, and covariates. a-d Depict the CWRES versus time after dose, the population predictions, renal function (eGFR) and actual body weight, respectively. Black open circles represent

strongly correlates to body -size. Using the BW-adjusted, rather than BSA-adjusted [9] eGFR, allowed separation of a renal and non-renal fraction of clearance. In addition, the estimated slope now represents the fraction of eGFR accounting for renal clearance. This slope was estimated at 0.78 , implicating that renal resorption of fludarabine occurs; however. this is based on the assumption that eGFR accurately reflects actual glomerular filtration rate. Furthermore, the use of the Schwartz and Cockroft-Gault equations, as well as the age-dependent capping of eGFR, may have impact on the relationship between actual glomerular filtration rate and eGFR. As seen in the VPC, the maturation of renal function might not be properly accounted for in the current model, thus careful monitoring might be advised for the very young children. the CWRES values and the solid grey line is a local regression fit of these values. Dashed lines depict the zero-line. CWRES conditional weighted residuals, $e G F R$ estimated glomerular filtration rate

Interestingly, the fludarabine label [36] only indicates a dose reduction (up to $50 \%$ ) when eGFR is below $70 \mathrm{~mL} /$ $\min / 1.73 \mathrm{~m}^{2}$. Although this $50 \%$ dose reduction is supported by our findings, an eGFR below $120 \mathrm{~mL} / \mathrm{min} / 1.73 \mathrm{~m}^{2}$ has already been associated with a substantial decrease in fludarabine clearance ( $>25 \%$ ) and concomitant high exposures. Furthermore, the decrease in clearance is a gradual process. Therefore, a dosing algorithm that takes eGFR into account, similar to the clinically applied algorithm for carboplatin, may be more appropriate for fludarabine. Such an algorithm could be an equation directly derived from the model-predicted clearance.

The applicability of such an algorithm depends on the diversity of the underlying dataset, which was sufficient in our study regarding age and indication, but less sufficient regarding co-conditioning agents (busulfan, \pm clofarabine). 


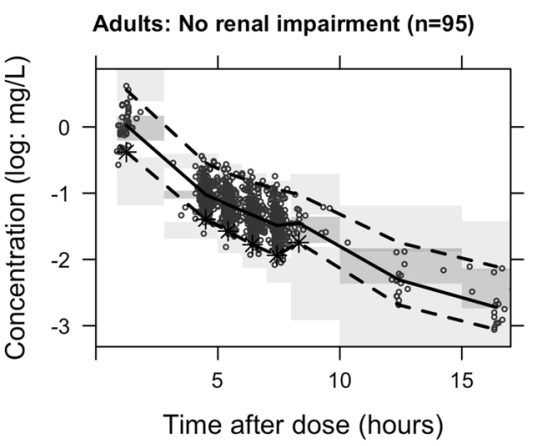

Children: No renal impairment $(n=115)$

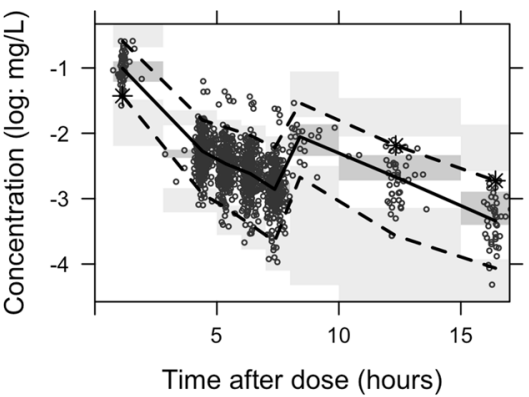

Adults: Mild renal impairment $(n=22)$

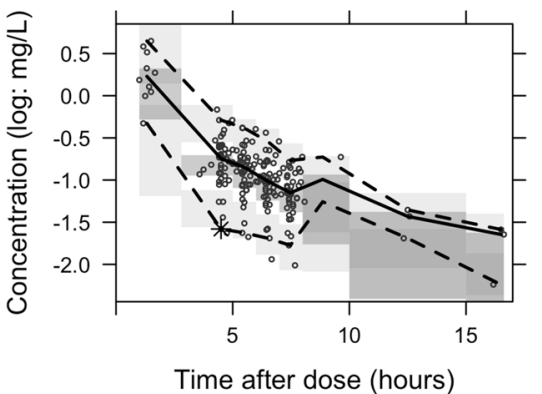

Children: Mild renal impairment $(n=15)$

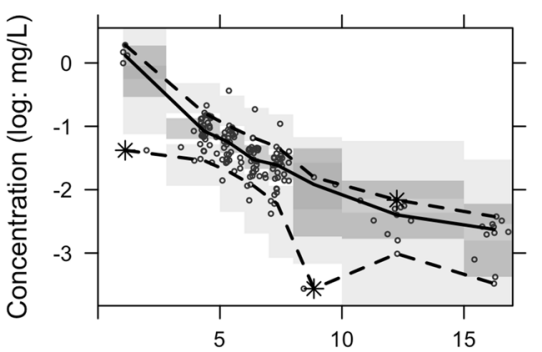

Time after dose (hours)
Adults: Moderate renal impairment $(n=7)$

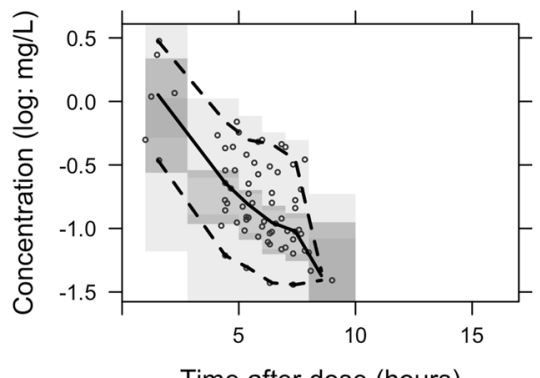

Time after dose (hours)

Children: Moderate renal impairment $(n=4)$

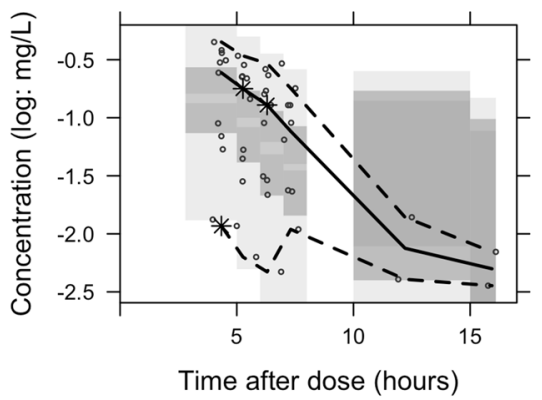

Fig. 4 Stratified prediction-corrected visual predictive check. Black lines depict the observed median (solid) and 2.5\% and 97.5\% percentile (dashed) concentrations. Dark- and light-grey areas represent $95 \%$ prediction intervals of the simulated mean and the 2.5 and $97.5 \%$ percentiles, respectively. Round dots represent observations. Asterisks

This has the disadvantage of not being able to quantify possible PK interactions with a variety of conditioning agents. Given the predominant renal elimination, such interactions are unlikely and were indeed found to be absent with clofarabine in these data. PK extrapolation of dosing algorithms may therefore be well translatable to other conditioning regimens, although the target exposure might differ. The advantage of this homogeneously treated cohort is the possibility of finding an optimal PK exposure for fludarabine in the widely applied busulfan/ fludarabine regimen.

\section{Conclusion}

Given the observed high variability in exposure, the current BSA-based dosing regimen, without taking eGFR into account, may not be appropriate. The current analysis provides a rational base for a harmonized optimal dosing regimen for all age groups in HCT.

Acknowledgements This work was supported by Foundation Children Cancerfree (KiKa) project number 190. highlight observed percentiles outside the prediction area. Increased median concentrations after $8 \mathrm{~h}$ are caused by the prediction correction. Subjects for whom samples for these bins were available had higher actual body weights

\section{Compliance with Ethical Standards}

Conflict of interest Jurgen Langenhorst, Thomas Dorlo, Erik van Maarseveen, Stefan Nierkens, Jaap Jan Boelens, Charlotte van Kesteren, and Alwin D.R Huitema have no conflicts of interest to declare. Jürgen Kuball receives research funding from, and is Chief Scientific Officer and shareholder of, Gadeta (www.gadeta.nl).

Open Access This article is distributed under the terms of the Creative Commons Attribution-NonCommercial 4.0 International License (http://creativecommons.org/licenses/by-nc/4.0/), which permits any noncommercial use, distribution, and reproduction in any medium, provided you give appropriate credit to the original author(s) and the source, provide a link to the Creative Commons license, and indicate if changes were made.

\section{References}

1. Gyurkocza B, Sandmaier BM. Conditioning regimens for hematopoietic cell transplantation: one size does not fit all. Blood. 2014;124(3):344-53.

2. Ben-Barouch S, Cohen O, Vidal L, Avivi I, Ram R. Busulfan fludarabine vs busulfan cyclophosphamide as a preparative regimen before allogeneic hematopoietic cell transplantation: systematic review and meta-analysis. Bone Marrow Transplant. 2016;51(2):232-40. 
3. Rambaldi A, Grassi A, Masciulli A, Boschini C, Mico MC, Busca A, et al. Busulfan plus cyclophosphamide versus busulfan plus fludarabine as a preparative regimen for allogeneic haemopoietic stem-cell transplantation in patients with acute myeloid leukaemia: an open-label, multicentre, randomised, phase 3 trial. Lancet Oncol. 2015;16(15):1525-36.

4. McCune JS, Mager DE, Bemer MJ, Sandmaier BM, Storer BE, Heimfeld S. Association of fludarabine pharmacokinetic/dynamic biomarkers with donor chimerism in nonmyeloablative HCT recipients. Cancer Chemother Pharmacol. 2015;76(1):85-96.

5. Plunkett W, Huang P, Gandhi V. Metabolism and action of fludarabine phosphate. Semin Oncol. 1990;17(5 Suppl 8):3-17.

6. Consoli U, El-Tounsi I, Sandoval A, Snell V, Kleine HD, Brown W, et al. Differential induction of apoptosis by fludarabine monophosphate in leukemic B and normal $\mathrm{T}$ cells in chronic lymphocytic leukemia. Blood. 1998;91(5):1742-8.

7. McCune JS, Vicini P, Salinger DH, O'Donnell PV, Sandmaier BM, Anasetti C, et al. Population pharmacokinetic/dynamic model of lymphosuppression after fludarabine administration. Cancer Chemother Pharmacol. 2015;75(1):67-75.

8. Woodahl EL, Wang J, Heimfeld S, Sandmaier BM, O'Donnell PV, Phillips B, et al. A novel phenotypic method to determine fludarabine triphosphate accumulation in T-lymphocytes from hematopoietic cell transplantation patients. Cancer Chemother Pharmacol. 2009;63(3):391-401.

9. Ivaturi V, Dvorak CC, Chan D, Liu T, Cowan MJ, Wahlstrom $\mathrm{J}$, et al. Pharmacokinetics and model-based dosing to optimize fludarabine therapy in pediatric hematopoietic cell transplant recipients. Biol Blood Marrow Transplant. 2017;23(10):1701-13.

10. Malspeis L, Grever MR, Staubus AE, Young D. Pharmacokinetics of 2-F-ara-A (9-beta-D-arabinofuranosyl-2-fluoroadenine) in cancer patients during the phase I clinical investigation of fludarabine phosphate. Semin Oncol. 1990;17(5 Suppl 8):18-32.

11. Salinger DH, Blough DK, Vicini P, Anasetti C, O'Donnell $\mathrm{PV}$, Sandmaier BM, et al. A limited sampling schedule to estimate individual pharmacokinetic parameters of fludarabine in hematopoietic cell transplant patients. Clin Cancer Res. 2009;15(16):5280-7.

12. Bonin M, Pursche S, Bergeman T, Leopold T, Illmer T, Ehninger $\mathrm{G}$, et al. F-ara-A pharmacokinetics during reduced-intensity conditioning therapy with fludarabine and busulfan. Bone Marrow Transpl. 2007;39(4):201-6.

13. Long-Boyle JR, Green KG, Brunstein CG, Cao Q, Rogosheske $\mathrm{J}$, Weisdorf DJ, et al. High fludarabine exposure and relationship with treatment-related mortality after nonmyeloablative hematopoietic cell transplantation. Bone Marrow Transpl. 2011;46(1):20-6.

14. Mohanan E, Panetta JC, Lakshmi KM, Edison ES, Korula A, Fouzia NA, et al. Population pharmacokinetics of fludarabine in patients with aplastic anemia and Fanconi anemia undergoing allogeneic hematopoietic stem cell transplantation. Bone Marrow Transpl. 2017;52(7):977-83.

15. Punt AM, Langenhorst JB, Egas AC, Boelens JJ, van Kesteren C, van Maarseveen EM. Simultaneous quantification of busulfan, clofarabine and F-ARA-A using isotope labelled standards and standard addition in plasma by LC-MS/MS for exposure monitoring in hematopoietic cell transplantation conditioning. J Chromatogr B Analyt Technol Biomed Life Sci. 2017;1055-1056:81-5.

16. Sheiner LB, Rosenberg B, Marathe VV. Estimation of population characteristics of pharmacokinetic parameters from routine clinical data. J Pharmacokinet Biopharm. 1977;5(5):445-79.

17. Keizer RJ, van Benten M, Beijnen JH, Schellens JH, Huitema AD. Pirana and PCluster: a modeling environment and cluster infrastructure for NONMEM. Comput Methods Programs Biomed. 2011;101(1):72-9.
18. R Core Team. R: A language and environment for statistical computing. R Foundation for Statistical Computing, Vienna, Austria. 2017. https://www.r-project.org/. Accessed 11 Oct 2018.

19. McCune JS, Bemer MJ, Barrett JS, Scott Baker K, Gamis AS, Holford NH. Busulfan in infant to adult hematopoietic cell transplant recipients: a population pharmacokinetic model for initial and Bayesian dose personalization. Clin Cancer Res. 2014;20(3):754-63.

20. Du Bois D, Du Bois EF. The measurement of the surface area of man. Arch Intern Med. 1915;15:868-81.

21. Ross SR, McTavish D, Faulds D. Fludarabine: a review of its pharmacological properties and therapeutic potential in malignancy. Drugs. 1993;45(5):737-59.

22. Cockcroft DW, Gault MH. Prediction of creatinine clearance from serum creatinine. Nephron. 1976;16(1):31-41.

23. Schwartz GJ, Haycock GB, Edelmann CM Jr, Spitzer A. A simple estimate of glomerular filtration rate in children derived from body length and plasma creatinine. Pediatrics. 1976;58(2):259-63.

24. Cohen E, Nardi Y, Krause I, Goldberg E, Milo G, Garty M, et al. A longitudinal assessment of the natural rate of decline in renal function with age. J Nephrol. 2014;27(6):635-41.

25. Krekels EH, van Hasselt JG, Tibboel D, Danhof M, Knibbe CA. Systematic evaluation of the descriptive and predictive performance of paediatric morphine population models. Pharm Res. 2011;28(4):797-811.

26. Hooker AC, Staatz CE, Karlsson MO. Conditional weighted residuals (CWRES): a model diagnostic for the FOCE method. Pharm Res. 2007;24(12):2187-97.

27. Byon W, Smith MK, Chan P, Tortorici MA, Riley S, Dai H, et al. Establishing best practices and guidance in population modeling: an experience with an internal population pharmacokinetic analysis guidance. CPT Pharmacometrics Syst Pharmacol. 2013;2:e51.

28. US FDA. Guidance for Industry, Population Pharmacokinetics. 1999. https://www.fda.gov/downloads/drugs/guidances/UCM07 2137.pdf. Accessed 11 Oct 2018.

29. EMEA. Guideline on reporting the results of population pharmacokinetic analyses. 2007. https://www.ema.europa.eu/documents/ scientific-guideline/guideline-reporting-results-population-pharm acokinetic-analyses_en.pdf. Accessed 11 Oct 2018.

30. Comets E, Brendel K, Mentre F. Computing normalised prediction distribution errors to evaluate nonlinear mixed-effect models: the npde add-on package for R. Comput Methods Programs Biomed. 2008;90(2):154-66.

31. Bergstrand M, Hooker AC, Wallin JE, Karlsson MO. Prediction-corrected visual predictive checks for diagnosing nonlinear mixed-effects models. AAPS J. 2011;13(2):143-51.

32. National Kidney Foundation. Glomerular filtration rate. 2018. https://www.kidney.org/atoz/content/gfr. Accessed 23 July 2018.

33. Rhodin MM, Anderson BJ, Peters AM, Coulthard MG, Wilkins $\mathrm{B}$, Cole $\mathrm{M}$, et al. Human renal function maturation: a quantitative description using weight and postmenstrual age. Pediatr Nephrol. 2009;24(1):67-76.

34. Anderson BJ, Holford NH. Mechanistic basis of using body size and maturation to predict clearance in humans. Drug Metab Pharmacokinet. 2009;24(1):25-36.

35. Germovsek E, Barker CI, Sharland M, Standing JF. Scaling clearance in paediatric pharmacokinetics: All models are wrong, which are useful? Br J Clin Pharmacol. 2017;83(4):777-90.

36. Fludara $50 \mathrm{mg}$ powder for solution for injection or infusion: Summary of Product Characteristics (SmPC)-(eMC). 2018. https:// www.medicines.org.uk/emc/product/1125/smpc. Accessed 14 May 2018. 